\title{
Taking an Early Step in Ethnobiological Research: A Proposal for Obtaining Prior and Informed Consent from Indigenous Peoples
}

\author{
Armando Medinaceli ${ }^{1,2^{*}}$ \\ ${ }^{1}$ Latin American Society of Ethnobiology (SOLAE), Mexico City, Mexico. ${ }^{2}$ Department of Anthropology, Washington State \\ University, Pullman, WA, USA. \\ *manduche@gmail.com
}

\begin{abstract}
Based on my own experiences from the field, in this paper I reflect on my work in Bolivia and Guatemala, collaborating with the Tsimane' and $Q^{\prime}$ eqchi' peoples, respectively. The aim of this reflection is to propose a set of guidelines for an early step in ethnobiological research. I understand an early step of research to be obtaining prior, informed consent of the peoples with whom we collaborate; a step I argue should be formalized and included as part of research proposals and documents (publications) resulting from the research. This guideline is offered simply as a reference for encouraging researchers to engage with the collaborating communities in a proper, ethical, and respectful way as a first step in our fieldwork. This proposed guideline, while motivating researchers to engage in this process, also encourages them to adapt and modify the guideline to the particular local situation where the planned research will take place. The proposal responds to local customs and traditions, while also following critical ethical guidelines for ethnobiological research, as well as national and international policy relevant to our field of research. It is therefore relevant to any region and community of collaborators where research takes place.
\end{abstract}

Received July 17, 2017

OPEN ӘACCESS

Accepted December 15, 2017

DOI 10.14237/ebl.9.1.2018.1054

Keywords Ethnobiological research, Prior and informed consent, Ethics, Policy

Copyright (c) 2018 by the author(s); licensee Society of Ethnobiology. This is an open-access article distributed under the terms of the Creative Commons Attribution-NonCommercial 4.0 International Public License (https://creativecommons.org/licenses/by-nc/4.0), which permits non-commercial use, distribution, and reproduction in any medium, provided the original author and source are credited.

\section{Introduction}

In this paper, I want to portray the importance of engaging communities in an appropriate and ethical way as a first step towards implementing an ethnobiological research/study. I begin by showing some of the legal and academic regulations and guidelines that offer ethnobiologists some tools to initiate this process, while also encouraging reflection on the importance of obtaining the prior and informed consent from the communities with whom we collaborate.

The Universal Declaration of Human Rights adopted by the UN's general assembly in 1948 provided regulations concerning equality for all peoples in the world. This declaration makes explicit the universality of human rights to all people, regardless of where people live and who they are (United Nations 1948).

By the late 1980s, the United Nations Environment Programme, in response to issues regarding the protection and conservation of biological diversity, developed the Convention of Biological Diversity (CBD). It was finalized in 1992 and put into practice by 1994. This convention is relevant to ethnobiological and anthropological research since it reflects issues related to the conservation of the world's biodiversity while supporting and allowing traditional and cultural practices and uses of the resources (Articles $8 j$ and 10c) by indigenous peoples and local communities (United Nations Environmental Programme 2003).

By the 2000s, nations of the world were engaged in a continuous discussion regarding indigenous peoples, the environment and climate. In Latin America, this represented a clear change in the politics of the region. Indigenous peoples became empowered, and for the first time had strong representation supported by the national governments of Brazil, Peru, Argentina, Bolivia, and Ecuador. 
Global discussions in the early 2000s resulted in the creation of the United Nations Declaration on the Rights of Indigenous Peoples (UNDRIP) in 2008. This declaration supports the rights of indigenous peoples to land and territory, and respect for their traditions, while also incorporating the principle of Free Prior and Informed Consent, and recognizing the self-determination of the indigenous peoples and local communities.

In 2010, the Nagoya Protocol on Access to Genetic Resources and the Fair and Equitable Sharing of Benefits Arising from their Utilization to the Convention on Biological Diversity emerged as a supplementary agreement to the CBD. The Nagoya Protocol commenced in 2014 (United Nations Environmental Programme 2017).

The paragraphs described above show some of the international agreements from a policy standpoint that regulate involvement of indigenous peoples and local communities. These policies therefore regulate academic research that involves the participation of indigenous peoples of the world. From an academic perspective, several examples of institutions and policies regulate anthropological and ethnobiological research.

In 1988 the International Society of Ethnobiology (ISE) was created, aiming to recruit ethnobiologists in support of the struggles of indigenous peoples and local communities while engaging in ethnobiological research. The creation of the ISE's Code of Ethics followed; a living document that offers guidelines for ethical approaches to ethnobiological research. This document is under constant discussion and review, which is discussed and approved in general assembly during their biannual conference (International Society of Ethnobiology 2008). The American Anthropological Association also offers ethical guidelines for anthropological research through its Code of Ethics (CoE) that was approved in 1998 and has its focus on anthropological research within the United States (American Anthropological Association 2012).

The Latin American Society of Ethnobiology (SOLAE) is the regional academic authority for ethnobiological research. SOLAE recently finished and approved its Code of Ethics in 2015. Its CoE aims to be a companion to the ISE's CoE while also touching upon issues that respond to the demands of indigenous peoples and local communities of the region.
All of these mechanisms are relevant to ethnobiological research. Use and respect of them should be encouraged within academia. An introduction to the policy relevant to our academic research should be incorporated at the university level, and when possible, some training, through examples, in the implementation of such mechanisms should be undertaken.

In the following paragraphs, I will describe my most recent experiences using policy and ethical mechanisms for the implementation of ethnobiological research with the Tsimane' in Bolivia and the Q'eqchi' in Guatemala.

\section{Obtaining Consent}

Obtaining Consent from the Tsimane' - Bolivia

With over 10 years of collaborating with the Tsimane' and by the time I was planning my current project, I was very familiar with the local traditions and customs and their social and political structure. In those years, I also learned more about "Western" resources available to me for research involving indigenous peoples, including the new Bolivian National Constitution (CPEPB 2009), Bolivian Law of the Mother Earth (Ley No. 300 2012), Universal Declaration of Human Rights (United Nations 1948), Convention on Biological Diversity (United Nations Environmental Programme 2003), United Nations Declaration on the Rights of Indigenous Peoples (United Nations 2008), and International Society of Ethnobiology code of ethics (ISE 2006). I also became involved in the work of creating a Code of Ethics for SOLAE.

This process started with visits to the communities where I intended to work. We discussed the research ideas and agreed on the topic of research. I received the authorization of the Tsimane' regional authorities. History shows that as a result of bureaucratic practices and corruption, encouraged by outsiders interested in their land and resources, Tsimane' authorities mostly grant permission to researchers engaging in quid pro quo relationships. In my case, in an attempt to break this type of relationship, I took a different approach. I engaged in a negotiation where I explained my proposed research (several times) and my approach based on co-inquiry, collaboration and following indigenous epistemologies. Thus, I was aiming for a research that would be beneficial for the villages, the regional authorities, and myself. 
In engaging in these consent discussions, I highlighted the ethical guidelines I was following (ISE's and SOLAE's codes of ethics), while also demonstrating that I was attempting to respond to international regulations such as UNDRIP, as well as national laws that favor indigenous peoples such as the 'Tsimane' (National constitution of Bolivia [CPEPB 2009], and Law of the mother earth, Bolivia [Ley No. 300 2012]).

After long negotiations (several meetings within a month), we reached an agreement in which I was granted their consent and permission to implement my research. This agreement included a set of regulations about the rights and responsibilities of all people involved in the research, respect for local traditions, respect for local scheduling, and other considerations. Also, I agreed to ask for consent from each participant, ask the parents for consent for the participation of children, and perform periodical presentations of the project's progress to the regional authorities and the participating villages, at their request. The agreement also forbade my consumption of alcoholic beverages while working on my research. Additionally, I agreed to train the authorities on computer use and grant writing, and I contributed by writing proposals, official letters, etc.

To seal the agreement, I offered a document to be signed, but as they said:

Us the Tsimane' always create oral agreements among ourselves, and we trust one another, that is how we agree on things, by talking. But if somebody does not follow the agreement we will also punish that.

Therefore, this process concluded with an oral agreement that remains valid until the results of the research are returned and approved by the villages and authorities. Finally, it was decided that we would make the agreement a live document, in order to make changes or additions, if needed.

While implementing my research, I organized meetings where we would discuss the possible formats in which the results from my research would have a better impact and be more locally beneficial. We agreed on a video documentary, possible booklet for school use, copies of published papers and their translation into Spanish when needed, the creation of a mobile exhibit, and presentation of results at regional and international congresses to share our experience of collaboration.
The process to obtain permission and consent in total took approximately four weeks. Even though this seemed like a long time, it created a stronger bond between the Tsimane' and me, and strengthened our friendship and trust. The process was incredibly enriching for me and my research, and also demonstrated to the Tsimane' that academic research can be respectful, sensitive, and beneficial to all parties involved. The Tsimane' benefited from the return of the results from my research in the formats I mentioned above which are considered relevant and locally useful. As a researcher, I benefited from the publications of scientific articles, making public presentations to share this collaborative experience, and from strengthening the relationship with the Tsimane' people, which helped further my research interests in the region.

\section{Obtaining Consent from the Q'eqchi' - Guatemala}

In 2016, I engaged in research in Guatemala, a new environment and culture for me as a researcher. For this project, I was not able to organize a previous visit to the village where I intended to work, so I contacted a Guatemalan colleague familiar with the village and with years of experience working in the region. With his help, I could introduce, in a letter, my idea and intention to collaborate with one Q'eqchi' village.

Once they accepted my proposal to work in their village, I organized my visit. In advance, I studied the national regulations, local traditions, and social and political structures of the Q'eqchi'. Upon arrival to the village, I had a meeting with the local authorities. In the meeting I introduced myself, and explained my intentions and the proposal in more detail. I asked for their permission to implement my research, making sure to explain clearly all details of what my research would entail; my collaborative approach, the methods to be used, and my intention to include children as participants.

Once we held the meeting, I was asked to present all points of our agreement in written format. I presented the document to the authorities and left the village for three days to give them space to review and discuss the points discussed for the agreement. On my return, I presented the final version of the document and they granted me permission and we signed the agreement. I was asked to present, once more, the proposal and whole idea of research to the general assembly of the community. 
Our agreement includes 12 points, highlighting the rights of people to choose to participate or not as part of my study; consent to carry out activities with children within the school compound during regular school hours; commitment to return results of the research in formats predetermined in discussion with villagers, as well as new ones that could emerge during the research; agreement that researchers are not allowed to consume any alcoholic beverages while conducting fieldwork or any activities related to the research and the duration of the agreement spanning until all results from research are returned and accepted by the villagers and authorities. As part of my commitment of contributing to the community, we agreed on my contribution to local initiatives, for which I gave a training session on proposal and grant writing. I also contributed to the writing of a proposal for a community-led project regarding the sustainable management of cacao. All processes of authorization and agreement took seven days.

During fieldwork, I had meetings and discussions with villagers about the possible formats for the results from this research, making sure that they become useful and have a positive impact on the whole village. We agreed to the creation of posters to be displayed in the village and at the local school, a booklet about the local uses of plants and animals to be presented as a complementary resource for school activities, and Spanish translations of any publication resulting from research.

\section{Making Connections}

Both experiences of obtaining permission and consent for implementing my research were based on international legal regulations, codes of ethics for ethnobiological research, and recognizing and respecting local customary regulations and formats for the creation of agreements and granting permits.

Both processes demonstrate that including such procedures as the first step in preparation of any study is extremely beneficial for both researcher and 'collaborators'. Benefits for the villages may include their empowerment by getting involved in decision making processes, such as deciding to take the research in a direction that is locally important and relevant; suggesting and contributing to the decision of the types, formats, and uses of the information resulting from research, including the possibility for co-authorship of publications. This process creates a sense of real collaboration and ownership of the research. Locals understand that they have the authority to regulate and control research activities, and this supports the self-determination of their villages. It also helps them feel more comfortable and engaged with academic research, as long as the agreements are fully respected and accomplished.

For the researcher, the benefits include creation of research that is academically and scientifically innovative (through a truly collaborative approach), research publications (that could include coauthorship with indigenous or local peoples), public presentations to distinct fora (academic or not) that are supported by the villages where research took place, a bonding experience with locals that ensures future collaborations and access to continue research activities on the region. Overall, with this approach I aim to ensure short and long term benefits for all parties involved.

As part of the collaboration and search of mutual benefits, reciprocity becomes an important aspect. Reciprocity ensures true collaboration and mutual benefit. It can involve tasks or negotiations that are not necessarily directly related to the research, but can be of mutual benefit. For instance, when in the agreement created with the Tsimane', I agreed to contribute to the regional authorities and local communities by offering training sessions on computer use, and on proposal and grant-writing for their own local research initiatives or development projects. I was also asked to collaborate in the writing and review of the resolutions from community meetings, and to offer free transportation to villagers any time I came and went to the villages. In exchange, in the reciprocity context, villagers would offer help as guides without any charge for forest excursions or visits to other villages. In the case of the Q'eqchi' village in Guatemala, I was asked to contribute my knowledge and skills in the elaboration of a proposal for a locally managed project regarding cacao production, while discussing and explaining the process of elaboration of a proposal. As part of the reciprocal arrangement, my research partner and I were granted lodging in the village at no cost throughout the duration of fieldwork. The benefits of reciprocity can be seen from multiple standpoints. From an academic stand point, communities commit to participate and contribute as much as possible to the research. From a logistical standpoint, lodging or other arrangements and costs may be agreed upon. From a community perspective, communities benefit not only from the results from research, but also in 
other aspects, such as training in computer use, grant writing, or other applicable skills.

This prior informed consent should be included in research education and training at universities, not only because it is beneficial and respectful, but also because in many cases is required by the law or international treaties. Therefore, as academics, we should respect and follow these guidelines and make explicit in our research the approach we used to obtain permission and consent.

\section{How to Engage Ethically with Indigenous Peoples}

With my growing interest in the ethics of ethnobiological research and my strong commitment to respecting customs and traditions of the local communities with whom I collaborate, I realized that, during all my years as a student and participating in several projects, I have not had any kind of formal guidance regarding how to engage the people with whom I want to collaborate. Feeling somehow unprepared has encouraged me to focus on finding the best, most appropriate way to engage with local people being respectful of their own norms while also being able to share and transmit my own message to create a space of real collaboration between researcher(s) and collaborators.

Following the experiences presented above (and others from past work), I came to realize that even though I was collaborating with different indigenous groups in distinct geographical regions, each with their own set of traditional norms and regulations, the consent agreements I reached basically followed a similar structure.

This represents an opportunity to utilize those experiences of engaging communities to obtain their consent and permission to implement a research project, and organize them into one format that summarizes all aspects discussed and agreed between myself, as the researcher, and the communities, as collaborators. Therefore, I decided to create a set of guidelines that has helped me when initiating my past and current research projects. I present the guidelines here with the hopes that it will help other ethnobiologists as a baseline for the incorporation of this process as a first step to ethnobiological research.

While not a definitive recipe or set of instructions, this guideline is intended for researchers (especially first time fieldworkers or early career ethnobiologists) as a reference scheme, which can be adapted and modified to make it appropriate and accepted locally, always considering the local norms and customary regulations. This approach will facilitate the daunting experience of having to figure out how to adequately approach a group of people in the most respectful and ethical manner.

\section{A Guideline for Obtaining Consent and Permission for Fieldwork}

Every time I start a new research project/study, I made it a norm for myself to begin by creating a formal agreement with the village(s) with whom I will collaborate. This consent agreement is based on their traditional norms and regulations while also responding to guidelines and regulations by which, as an ethnobiologist and a responsible researcher, I should abide. Regulations such as the Codes of Ethics from ISE and SOLAE, but also UNDRIP, CBD, and others. This is the early first step to my research.

I came up with a format that encompasses the different consent agreements I created in the past collaborating with different indigenous peoples of Latin America. Table 1 summarizes the proposed format, but in the following paragraphs I discuss briefly the basic structure of my proposed guidelines.

\section{Terms and Conditions}

In all cases, when starting to discuss the creation of an agreement between myself (or team) as the researcher, and the villagers (authorities, representatives, etc.) as the collaborators of the research, we discuss the terms and conditions of the agreement. Explaining clearly what is entailed as part of the agreement, and as much as possible framing the agreement within the activities that will be considered part of the research. Terms and conditions include a detailed explanation of the duration of research, making sure to explain that research can change in duration because of unforeseeable factors. Also, it is important to make clear that some activities not planned originally can become a component of the research. This component of the agreement also includes a statement of "revision", meaning that the agreement can be revised and modified as it becomes necessary. This revision should always be with the authorization and agreement of all parties involved in the research.

\section{Research Summary}

Usually when meeting with collaborators, a presentation of the research proposal should take place. This presentation should be a detailed 


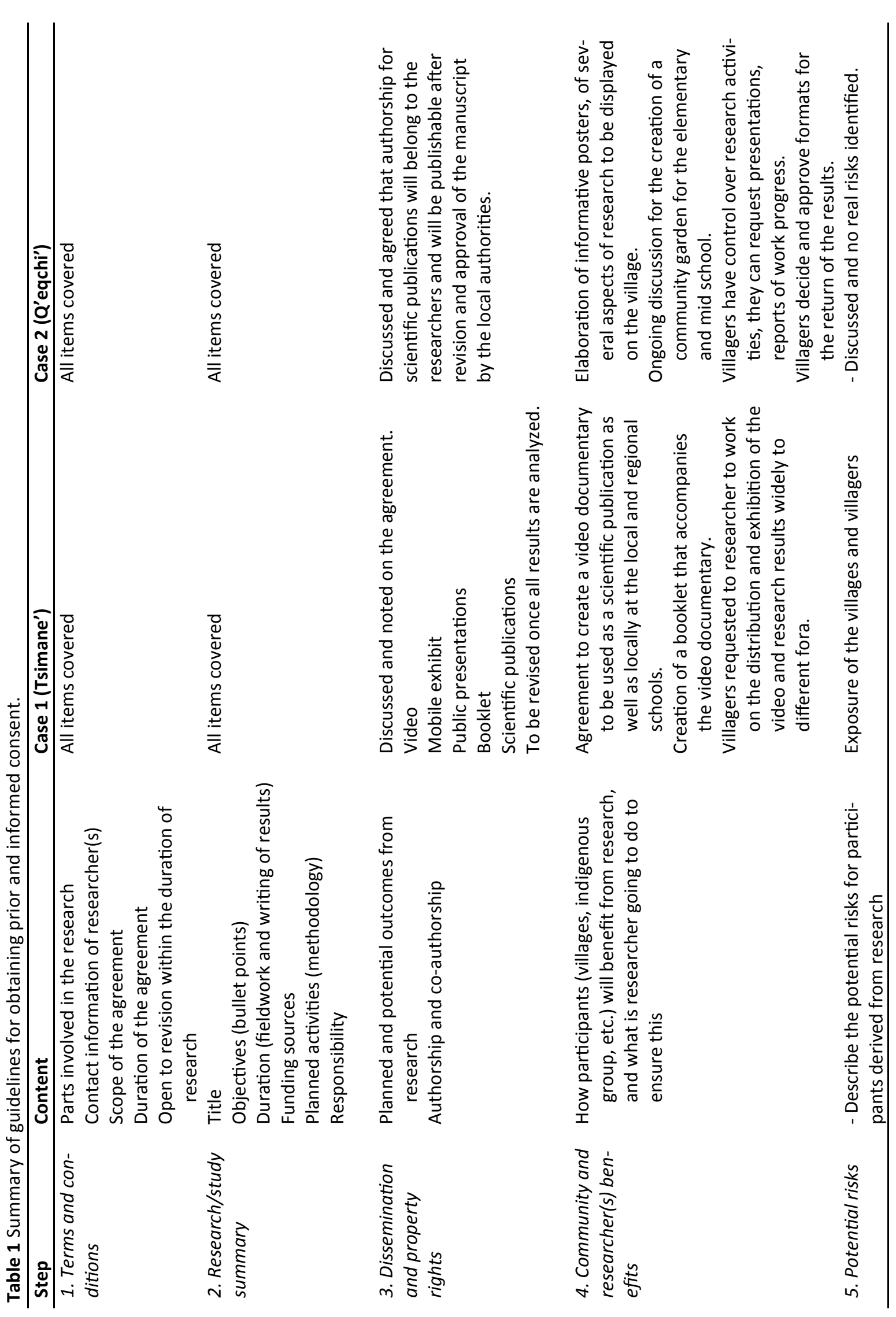




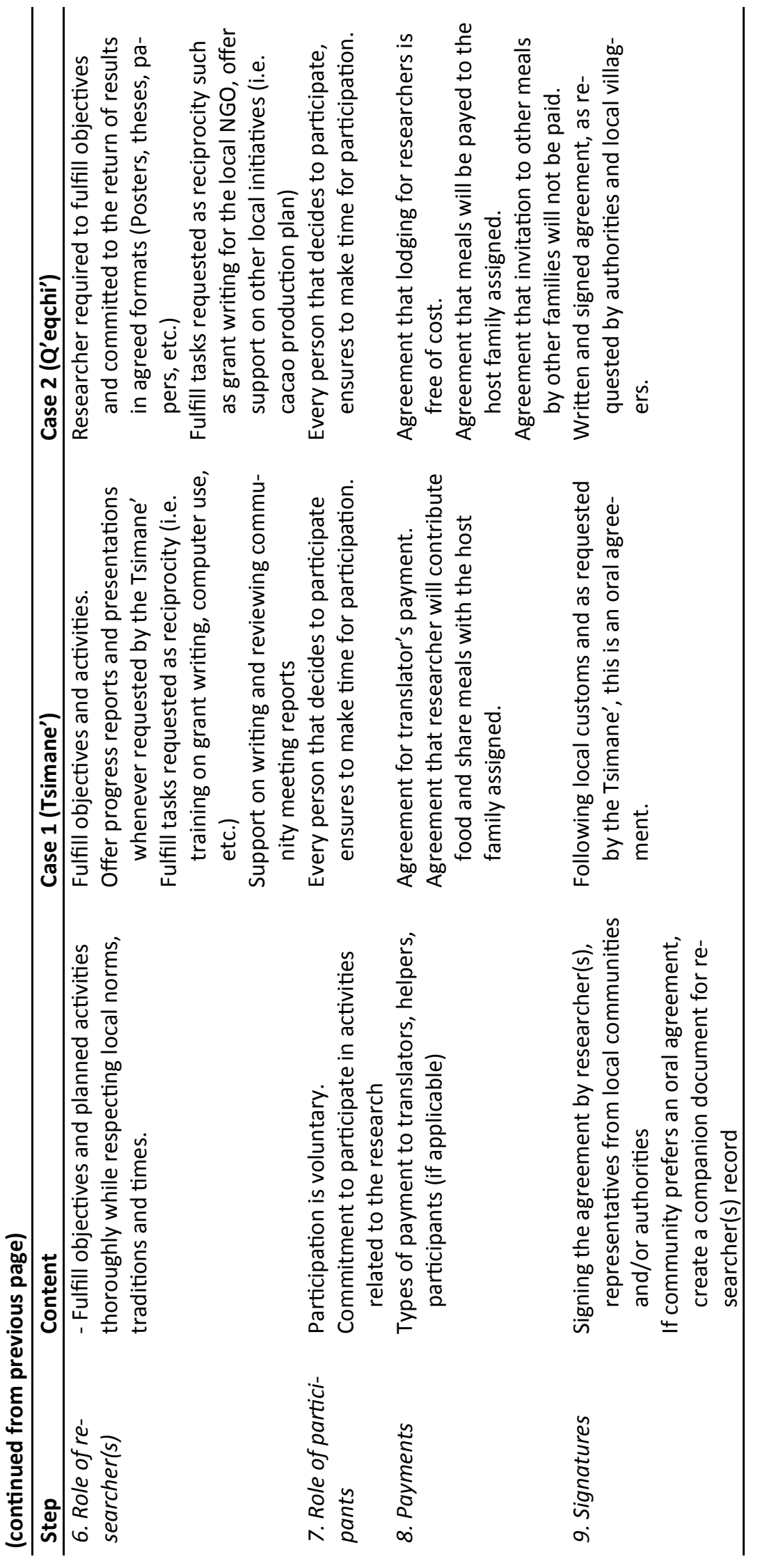


explanation of all activities that will take place, and the commitment expected from all participants. Depending on where the research will take place, at times the use of simpler language or a translator will be required to make sure that the project is completely understood.

This presentation will become the summary of the research as a second component of the agreement. A presentation and discussion of the research at the beginning of fieldwork is not enough. I believe that having a detailed written summary of the research within the consent agreement contributes to avoiding doubts or concerns about activities or components of research while fieldwork takes place. In addition, it gives collaborators the opportunity to have a tool for reviewing, evaluating and controlling project progress, especially long-term projects.

When work will be carried out by a team of researchers, it is recommended to have information of the researcher who is going to oversee each activity/ methodology of the project, including contact information to facilitate and encourage communication between participants and researchers.

\section{Dissemination, Property Rights, Benefits, and Risks}

When discussing and presenting the research with communities, a couple of topics often arise. The first one can be summed in the following question: What are the benefits that the proposed research project will bring to the participants, the villages and/or the region? This is a recurrent question that we, as researchers, should ask ourselves when creating our proposals.

Usually researchers have a clear understanding of the benefits of their research, but that does not necessarily imply what the benefits will be for the local communities. I believe that it is important to think in advance about what the research will bring to the local people and communities.

To answer this question, we must be careful and honest with our own views. An ideal research project should be beneficial to both the researcher(s) and the collaborators (local communities), but the benefits for each may be vastly different. Thus, explaining and discussing the possibilities should be an important component of the consent agreement (for more on benefits, refer to the examples presented above).

The second topic to which we must respond is: what is going to happen with all the information resulting from the research? It should also be mentioned if the resulting information will be taken away, published, or returned to the local context, and if so, in what formats? This is an issue that should be open to discussion. It is known that while carrying out research, it often happens that we (researcher and collaborators) realize that certain components or preliminary results can be beneficial for either one of the participants. Therefore, the consent agreement should maintain this component as open, i.e., with some initial expected goals (e.g., publications and books), but open to discussion and revision based on new priorities (e.g., responding to current situations and conflicts) that might arise during the implementation of research.

Talking about the benefits of research is just one side of the coin; the other side, often not well appreciated, involves the potential risks that a particular research project can bring to the local communities and collaborators, as well as the researchers. Therefore, consent agreements should have a section where risks can be made explicit. This will potentially alleviate any problems that could arise simply due to the implementation of research. This is an honest approach accepting that not every project is completely harmless, or that it is almost impossible to foresee what the research could offer or cause.

\section{The Role of Everyone Involved}

When starting my first independent projects as an undergraduate student, at times I experienced a lack of participation and commitment of some of my collaborators, especially when invited to meetings or other activities. At times, they just skipped an activity because they were not fully aware of their role and responsibilities within the project, and also some collaborators were not aware of my own commitment and responsibilities within the project. Therefore, for future projects I started discussing explicitly the nature of responsibilities I have to the research, the local people (respecting their traditions, customs), and the kind of commitment expected from participants (researcher and collaborators), even though their participation is voluntary.

Levels of involvement and participation in a project usually vary widely, starting from funders who do not really get involved with fieldwork, to the researchers who are fully involved and in charge of every step of the research process, to local participants/collaborators that will participate in some activities during fieldwork, and others that will possibly be involved in the complete process, such as 
key informants, translators, communal authorities, etc. Making clear all these aspects of research is important, because it ensures the respect and commitment of everyone who decides to get involved in the research process.

\section{Payments, Signatures}

Depending on the type of research and according to what is accepted within the local context, at times people will receive payments, either for participating in the research or for offering other services (e.g., food, translation, and guides). Any type of payments should be expressed in the consent agreement to avoid potential conflicts with and between paid and unpaid participants. When research/studies do not involve any type of payment or compensation to participants, it also should be stated clearly, once more to avoid any potential conflict.

Finally, once more following local regulations and to formalize the consent agreement, the locally accepted format for the agreement is the recommended one to follow. In my own experiences, most people then demand a written and signed agreement. But other formats that are locally and traditionally accepted also give validity to the agreement, such as oral agreements. When participants/collaborators acknowledge that an oral agreement is the appropriate and traditionally accepted format for providing consent, then the agreement should follow this format.

\section{Conclusions}

To facilitate the use of this proposed format, Table 1 summarizes the structure of the consent agreement process. The agreement should follow the local norms and customs as much as possible, while also responding to any regional, national or international regulations that affect the implementation of the research/study.

As mentioned above, this format is simply an encouraging guide in engaging communities as a first step of our research, and not as a set format. Working in different geographical, cultural and political contexts, following just one format can become problematic, but having this guideline that aims to cover the basic topics to organize an acceptable consent agreement can be beneficial.

I encourage ethnobiologists, anthropologists, and any researcher to understand the importance of implementing the process of engaging communities as a formal step of their research, to make it explicit in their research proposals, as well as in their publications or any format that results from their research. I strongly believe that to make our research more ethical, we should include an explanation of the steps followed for obtaining the consent from people we collaborate with as part of any resulting publication, from scientific to non-scientific (books, posters, brochures) to Masters theses and $\mathrm{PhD}$ dissertations.

The use of this guideline should be preceded and accompanied at the very least with a detailed review of the Codes of Ethics from the International Society of Ethnobiology (ISE, 2006), and the Latin American Society of Ethnobiology (SOLAE, 2016).

\section{Declarations}

Permissions: None declared.

Sources of funding: None declared.

Conflicts of Interest: None declared.

\section{References Cited}

American Anthropological Association. 2012. Ethics

Statement. Available at: http:/ /

ethics.americananthro.org. Accessed on February 9, 2017.

Constitution Politica del Estado Plurinacional de Bolivia (CPEPB). 2009. UPS Editorial, La Paz Bolivia.

International Society of Ethnobiology - ISE. 2006. ISE Code of Ethics (with 2008 additions). Available at: http://ethnobiology.net/code-ofethics/. Accessed on January 24, 2017.

Ley No. 300. Ley Marco de la Madre Tierra y Desarrollo Integral Para Vivir Bien. 2012. Gaceta Oficial de Bolivia.

Nagoya Protocol on Access to Genetic Resources and the Fair and Equitable Sharing of Benefits Arising from their Utilization (ABS) to the Convention on Biological Diversity Available at: www.cbd.int/ abs/about/. Accessed on March 15, 2017.

SOLAE, Sociedad Latinoamericana de Etnobiología, 2016. Código de Ética. Etnobiología, 14(1). Distrito Federal, Mexico.

United Nations Environmental Programme (UNEP). 2003 Convention on Biological Diversity - Text and Annexes. Booklet. Montreal, Canada.

United Nations Environmental Programme (UNEP) 2017. Convention on Biological Diversity. 
Available at: www.cbd.int/. Accessed on March 14, 2017.

United Nations. 1948. Universal Declaration of Human Rights. Available at: http://www.un.org/ en/universal-declaration-human-rights/. Accessed on February 10, 2017.
United Nations. 2008. United Nations Declaration on the Rights of Indigenous Peoples. Available at: https://www.un.org/development/desa/ indigenouspeoples/declaration-on-the-rights-ofindigenous-peoples.html. Accessed on February 9, 2017. 